\title{
A Decrease of Unsuccessful Pairing Probability in VAMOS Mobile Cell Using Existing Connection Rearrangement
}

\author{
Aleksandar LEBL, Dragan MITIĆ, Žarko MARKOV, Mihailo STANIĆ, Mladen MILEUSNIĆ
}

\begin{abstract}
This paper analyzes the existing connection rearrangement in mobile telephony systems with implemented VAMOS techniques. The main objective of rearrangement application is to decrease connection unpairing loss when there is exactly one idle traffic subchannel (OSC) in the system. The rearrangement technique was well-known in old multistage switching systems, where it contributed to traffic loss decrease. It is proved in this paper that connection loss probability due to pairing inability is decreased when rearrangement is implemented. Connection realization is improved in following cases: when offered traffic is greater, when allowed emission base station power difference between two paired OSCs in channels is smaller and when greater channel groups are implemented. Results are verified by computer simulation and illustrated by a numerical example.
\end{abstract}

Keywords: pairing loss; pairing probability; rearrangement; VAMOS

\section{INTRODUCTION}

VAMOS technique is implemented in modern GSM cells to increase traffic capacity, i.e. to allow more connections realization. One limitation of this technique is inability to pair connections, whose downlink emission powers have too different intensity level, [1], [2]. The pairing inability is caused by too great co-channel interference from the paired user, because each user receives his signal and the signal of the paired user, [3]. Co-channel interference has often influence on the outage probability in mobile systems, as also, for example, in systems analyzed in [4]. As a consequence of pairing inability in systems with implemented VAMOS technique, the new loss type appears, i.e. the loss due to pairing inability. This loss is increased by the loss due to the absence of idle subchannels (OSC), thus making total loss greater than the losses in the classical fully available group. Besides, the maximum number of unsuccessful pairing happens when there is only one idle OSC. This paper shows how much the probability of unsuccessful pairing may be decreased, if existing connections rearrangement is realized in the simplest case, i.e. when there is exactly one idle OSC and when the condition is established for exactly one of the already paired users.

The survey of previous results related to the application of VAMOS techniques is presented in Section 2. Basic concepts and designations, as well as the initial model parameters, necessary for the analysis in this paper, are emphasized in Section 3. Sections 4 and 5 show baseline of pairing (Section 4) and rearrangement (Section 5 ), as the processes of VAMOS connection realization. Section 6 presents mathematical model which is designed to estimate unsuccessful pairing probability decrease as a consequence of rearrangement implementation. Presented model is illustrated by a numerical example in Section 7 . An implemented simulation program is briefly described in Section 8. At the end, Section 9 is the conclusion of the paper.

\section{EXISTING SOLUTIONS}

Available literature, considering VAMOS systems, is not numerous. But, nevertheless, this technology is applicable to a significant extent in realized mobile systems, [5], [6], with the main goal to increase traffic capacity. Approximately, VAMOS techniques implementation allows traffic capacity duplication when full rate VAMOS channels are applied, and it allows traffic capacity quadruplicating when half rate VAMOS channels are applied. A more precise analysis, which considers Erlang traffic model, is presented in [7] and illustrated in [8]. The results from these contributions prove that increase in traffic capacity due to user pairing is greater than the approximately estimated increase, when influence of traffic is considered. Different types of user pairing are explained in [9], [10]. As for the applied order in seizing a completely idle channels and connection pairing in the channel with only one busy OSC, there are two pairing strategies, as it is emphasized in [11]: the first one is to pair all users, who may be paired (pure VAMOS) and the second one is to start pairing when some threshold of the number of busy channels without VAMOS implementation is reached. VAMOS implementation decreases mean necessary power per one active user, as it is analyzed in [12]. Although [12] is comprehensive consideration of user pairing, it does not explicitly emphasize some factors, which have influence on the pairing possibilities. These factors (user distance from the base station, user distribution in the base station cell and environmental attenuation factor $(\gamma)$ ) are indirectly analyzed (or, even, not analyzed). Rearrangement as a method of traffic resources efficiency improvement is analyzed in [13], where it is proved that the implementation of this method decreases total traffic loss. Two possible strategies of rearrangement with their advantages and disadvantages are presented in [14].

Generally speaking, implementation of various techniques for power saving in mobile communications systems is important and, even, mandatory request. Besides contribution to environment protection over the program of energy saving, called GREEN Radio, [15], 
power control in mobile systems contributes to its more quality functioning, [16].

\section{MODEL, ASSUMPTIONS AND DESIGNATIONS}

In this model, mobile cell is circular, with radius $R$. The control of emission power is implemented in a base station (BS) in the direction $\mathrm{BS} \rightarrow$ MS. The distance between MS and BS is $r, 0 \leq r \leq R$. Signal attenuation is designated by $a$. To make model simple, it is considered that the received signal attenuation depends on the distance between MS and BS, $a=k_{a} \cdot r^{\prime}, k_{a}$ is coefficient of proportionality and $\gamma$ is attenuation coefficient $2 \leq \gamma \leq 5$, [17]. The number of MSs is $M$ and the number of implemented traffic timeslots (traffic channels, TCH) is $N$. VAMOS techniques is implemented, i.e. each timeslot may be used by two telephone connections over two orthogonal subchannels (OSCs). Traffic (sub)channels are mainly used for telephone calls realization, which means that the request for a new connection that appears in a random moment must be realized in a short time interval, [18], [19]. Total offered traffic $(A)$ has constant intensity i.e. does not depend on system state. Service time, i.e. connection time, is random variable with negative exponential distribution. The mean connection time is $t_{m}=1 / \mu, A=\lambda / \mu=\lambda \cdot t_{m}$, and $\lambda$ is call intensity. It is supposed that pure $V A M O S$ model is implemented, [1], where all active subscribers use VAMOS techniques, i.e. the maximum attainable number of connections is $N$.

As the majority telephone serving systems, this one is also with loss. The only difference of this model comparing to Erlang model of full availability is in the case of pairing inability. When pairing is not possible, availability is limited, i.e. each user cannot seize each idle OSC. State probabilities in the considered VAMOS model are designated by $P\{j\}, j=0,1, \ldots, 2 N-1,2 N$, and the probability that all channels are busy is presented as $P\{2 N\}$. Pairing probability without rearrangement is $P u$, and with rearrangement $P r$. The probability of pairing inability is $P n$. Loss due to the lack of idle channels is designated by $B=P\{2 N\}$. Difference in power, which is applied to users with different loss, is designated by $\Delta$. The BS emission power to MS is designated by $P$, where $P_{\min }$ $\leq P \leq P_{\max }\left(P_{\min }\right.$ is the emission power, sent to the nearest users and $P_{\max }$ is the power, sent to the furthest users). Power control in BS is realized in 15 steps of $2 \mathrm{~dB}$ each, as represented in Fig. 1, [20], [21]. The cell part (ring), where the same power is intended for all users, is called area and designated by $a 1, \ldots, a 15$. The probability that MS belongs to the area $a i$ is designated by $p_{i}, i=1, \ldots, 15$.

\section{PAIRING}

Pairing is process of a new telephone connection realization using idle OSC in one timeslot, if the other OSC in that timeslot is already busy. The main condition of successful pairing is that necessary emission power difference for paired connections is smaller or equal to the limiting allowed power, $\Delta l$. In practice allowed difference of emission power may be $4,6,8$ or $10 \mathrm{~dB},[10]$, [22]. The main indicator of pairing effectiveness is (un)pairing probability. The successful pairing probability will be greater if $\Delta l$ is greater. In hypothetical case of $\Delta l$ increase towards value $30 \mathrm{~dB}$, pairing probability becomes close to 1 and the model becomes close to classical Erlang model. It is obvious that unsuccessful pairing probability is the greatest for the smallest value of $\Delta l$, i.e. for $\Delta l=4 \mathrm{~dB}$. Consequently, in focus of this research is the analysis how to decrease unsuccessful pairing probability when $\Delta l=4$ $\mathrm{dB}$.

\section{REARRANGEMENT}

The rearrangement is process of existing connection realization by new resources, and it is used in order to increase the traffic capacity. This technique is studied in the use of multistage switching in telecommunication centres, [23]. Using it, in order to realize new connections by currently engaged resources, a new path is looked for in a space multiplex of multi-stage switching system (rearrangeable networks) for the already established connections. Technically, rearrangeable networks in multistage switching systems are different from time channels in mobile cell, but the goal of rearrangement is the same in both cases.

The first step in existing VAMOS connection rearrangement is connection unpairing from individual traffic channels, followed by replacement of previously paired connection (seizing single channel) by two connections (each seizing entire traffic channel). This process of unpairing is called demultiplexing in [5]. The reasons for demultiplexing, according to [5], are quality impairment of one or both connections in an established pair and instantaneous traffic load decrease. In the case that after some time traffic load is increased again, demultiplexed connections may be grouped newly in VAMOS pairs, whereby the paired connections do not have to be re-established between the same two previously paired users, but completely new combinations of paired users may be formed. In contrast to [5], wherein the reduction of traffic load causes unpairing, and re-pairing may possibly occur if the traffic load increases later, this paper discusses a method of unpairing and, obligatory, repairing in order to prevent traffic loss of the newly created connection, which cannot be paired with an already established connection. Therefore, in principle, this paper analyzes the situation when by traffic load is increased, and the process of unpairing the existing VAMOS pair and a new pair forming is taking place one after the other, in a short period of time, without establishing a single connection in a channel.

\section{USING REARRANGEMENT TO DECREASE UNSUCCESSFUL PAIRING PROBABILITY}

Let us suppose that total unpairing probability is known. It includes the cases where there are $1,2, \ldots, N$ channels with unpaired OSCs, and there are no completely idle channels (with both idle OSCs), but allocation of busy OSCs in unpaired channels is such that new connection cannot be paired. The dominant influence on the unsuccessful pairing probability is related to the pairing attempt when there are $2 N-1$ realized connections, meaning that there is only one idle OSC in the system. In this paper is demonstrated how much is reduced the probability of unsuccessful pairing at the last idle OSC if 
connections rearrangement is applied in one timeslot to allow the seemingly impossible pairing. (Rearrangement procedure may be performed applying several signalization cycles, in a simpler way than in handover state, [20]. The prolonged time of connection setup due to rearrangement is negligible, i.e. imperceptible to the user.)

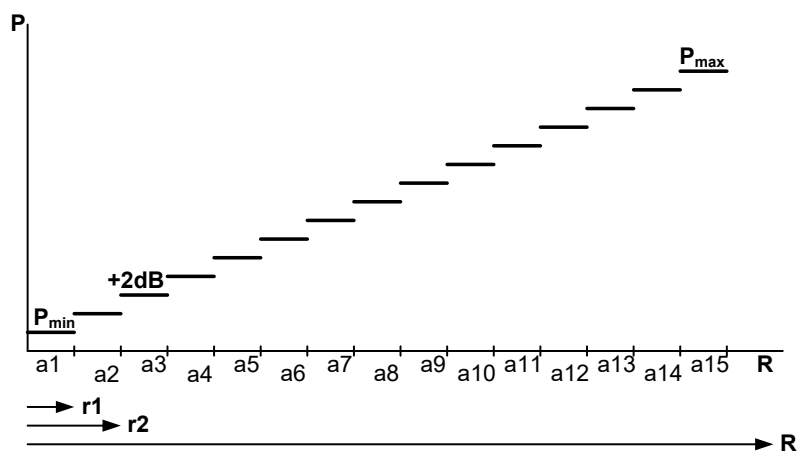

Figure 1 Illustration of power control in BS

Let us analyze a system where it is possible to pair users, whose necessary signal power difference is not greater than $4 \mathrm{~dB}$. The successful pairing probability in this case $(\Delta l=4 \mathrm{~dB})$ is equal to the probability that one call is from the area $a 4\left(p_{4}\right)$ and the probability that the other call is from the area $a 2, a 3, a 4, a 5$ or $a 6\left(p_{2}+p_{3}+p_{4}+p_{5}+p_{6}\right)$, i.e.

$P_{p(i=4)}=p_{4} \cdot\left(p_{2}+p_{3}+p_{4}+p_{5}+p_{6}\right)$

Let us examine a system in the state $\{2 N-1\}$ and the only one half-busy timeslot (Fig. 2). The OSC in this halfbusy timeslot is engaged by the user MS1 (triangle) who, according to distance from BS and necessary signal power, belongs to the ring 1 (area $a 1$ ). User MS2 - candidate for pairing (square) may belong to the ring $i$ with the probability $p_{i}(i=1,2, \ldots, 15)$. If the allowed necessary power difference for connections pairing is $\Delta l=2 \mathrm{kdB}=4$ $\mathrm{dB}$, these two connections may not be paired. If the power difference between these users is a bit greater than $4 \mathrm{~dB}$ (i.e. $6 \mathrm{~dB}$ or $8 \mathrm{~dB}$ ), pairing may be realized under one condition. It is necessary that at least one user (circle) from timeslots with paired connections is positioned in the rings between the considered ones. (square and triangle in this examination).

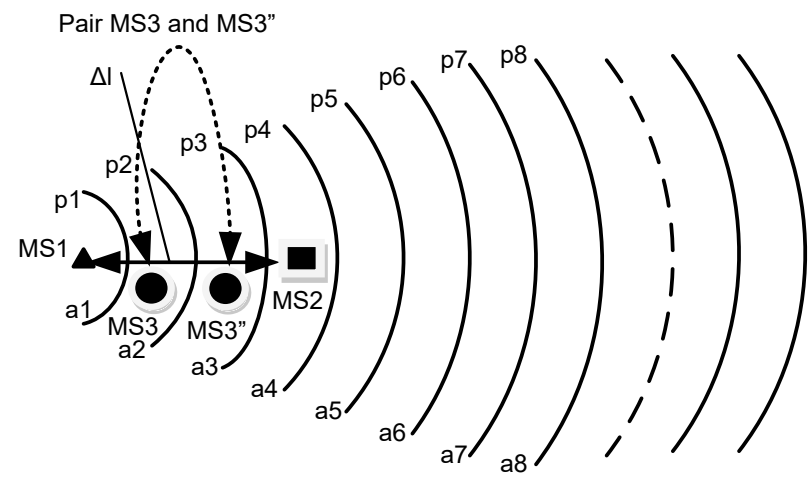

Figure 2 Illustration of conditions for possible connection pairing (MS1 and MS2), when necessary signal power difference is $6 d B$, i.e. greater than allowed value of $4 d B$
Fig. 2 presents the case that candidate for pairing (MS2) is located in the ring, which requires $6 \mathrm{~dB}$ greater emission power than the ring 1. Pairing is not possible without rearrangement of existing connections. If one of the users in paired connections (circle) is located in the ring 2 (MS3, it is paired with MS3"), then connection rearrangement is possible on the following principle: users MS1 and MS2 are paired with unpaired users MS3 and MS3" on the principle of approximate power: MS1 and MS3 are paired in one timeslot, and MS2 and MS3" in the second, remaining timeslot.

The same consideration is valid for an example in Fig. 3 if one of paired users belongs to the area $a 3$.

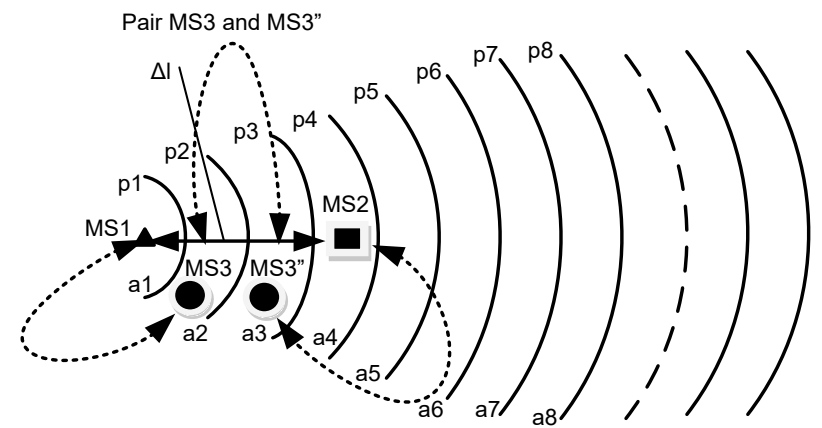

Figure 3 Existing connections rearrangement to allow pairing of user (MS2), who has necessary power difference in relation to unpaired user (MS1) $6 \mathrm{~dB}$, i.e. greater than allowed value of $4 \mathrm{~dB}$

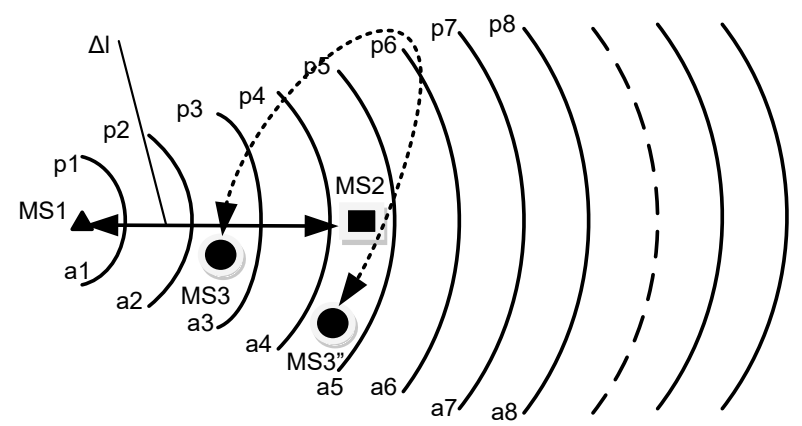

Figure 4 Illustration of conditions for possible connection pairing (MS1 and MS2), when necessary signal power difference is $8 \mathrm{~dB}$, i.e. greater than allowed value of $4 \mathrm{~dB}$

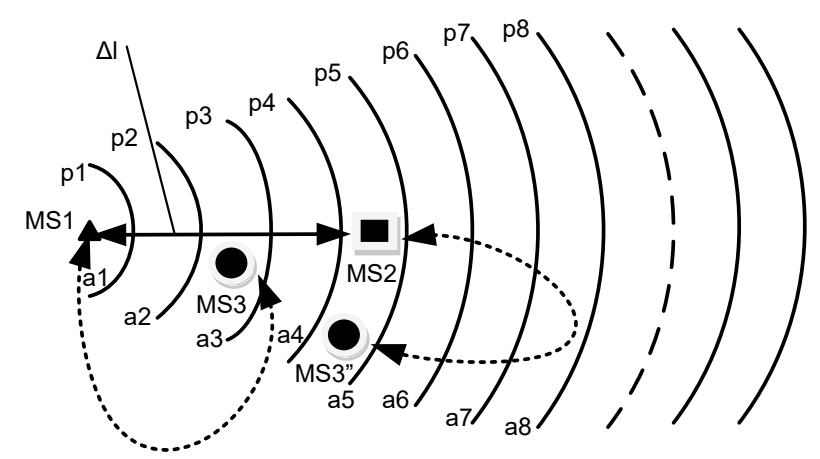

Figure 5 Existing connections rearrangement to allow pairing of user (MS2), who has necessary power difference in relation to unpaired user (MS1) $8 \mathrm{~dB}$, i.e. greater than allowed value of $4 \mathrm{~dB}$

Let us now consider the case when the difference of necessary power for users - candidates for pairing (MS1 and MS2) is $8 \mathrm{~dB}$, Fig. 4. Since there is a paired user (MS3 with the user MS3") located in a ring which is $4 \mathrm{~dB}$ away from candidates for a new pairing, Fig. 4, process of paring is realized in the following way: 
- the paired users MS3 and MS3" are unpaired,

- $\quad$ user MS1 is paired with MS3, and user MS2 with MS3", Fig. 5.

Pairing with rearrangement in which it is required that only one user in some connection is positioned in a ring between candidates for pairing will be called pairing with one rearrangement. In this paper only this type of rearrangement will be considered.

This case is analyzed from the probability view-point. The difference in the allowed emission power of paired users is $4 \mathrm{~dB}$. There are three possible outcomes when pairing is tried:

a) pairing is possible without rearrangement,

b) pairing is possible with one rearrangement,

c) pairing is impossible.

a1) Probability that the only unpaired OSC is in the area $a 1$ is $p_{1}$. Probability that direct pairing is possible in this case, i.e. that candidate for pairing is from the areas $a 1, a 2$ or $a 3$ is:

$P u(1)=p_{1} \cdot\left(p_{1}+p_{2}+p_{3}\right)$.

b1) Pairing with one rearrangement is possible if the new call is from the area $a 4$ or $a 5$. If the new call is from the area $a 4$, the probability that pairing is realized after rearrangement depends on the probabilities of the following events:

- $\quad$ the new call is from the area $a 4\left(p_{4}\right)$ and

- in realized connections at least one user is from the area $a 2$ or $a 3\left(p_{2}+p_{3}\right)$. There are $2 N-2$ users in paired connections. The probability that one paired user is not from the area $a 2$ or $a 3$ is $1-\left(p_{2}+p_{3}\right)$. The probability that no one of $2 N-2$ users is from the area $a 2$ or $a 3$ is

$$
\left[1-\left(p_{2}+p_{3}\right)\right]^{2 N-2}
$$

The probability that at least one of paired users is from the area $a 2$ or $a 3$ is:

$$
\left\{1-\left[1-\left(p_{2}+p_{3}\right)\right]^{2 N-2}\right\}
$$

and the probability that pairing may be realized after one connection rearrangement is:

$$
\operatorname{Pr} 1(1)=p_{1} \cdot\left(p_{4}\right) \cdot\left\{1-\left[1-\left(p_{2}+p_{3}\right)\right]^{2 N-2}\right\} \text {. }
$$

Let us now suppose that a new call is from the area $a 5$. Similarly as in previous case, pairing probability is

$$
\operatorname{Pr} 2(1)=p_{1} \cdot\left(p_{5}\right) \cdot\left\{1-\left[1-\left(p_{3}\right)\right]^{2 N-2}\right\} \cdot
$$

Pairing probability after one connection rearrangement, if unpaired user is from area $a 1$, may be calculated from:
$\operatorname{Pr}(1)=\operatorname{Pr} 1(1)+\operatorname{Pr} 2(1)$

c1) The probability of pairing inability is

$\operatorname{Pn}(1)=p_{1} \cdot\left(p_{6}+\ldots+p_{15}\right)$

$a 2$ ) If the unpaired user is from the area $a 2$, direct pairing probability is

$$
P u(2)=p_{2} \cdot\left(p_{1}+p_{2}+p_{3}+p_{4}\right)
$$

b2) The probability that pairing is realized after one rearrangement is

$$
\begin{aligned}
& \operatorname{Pr} 1(2)=p_{2} \cdot\left(p_{5}\right) \cdot\left\{1-\left[1-\left(p_{3}+p_{4}\right)\right]^{2 N-2}\right\} \\
& \operatorname{Pr} 2(2)=p_{2} \cdot\left(p_{6}\right) \cdot\left\{1-\left[1-\left(p_{4}\right)\right]^{2 N-2}\right\} \\
& \operatorname{Pr}(2)=\operatorname{Pr} 1(2)+\operatorname{Pr} 2(2) .
\end{aligned}
$$

c2) The unsuccessful pairing probability is

$$
\operatorname{Pn}(2)=p_{2} \cdot\left(p_{7}+\ldots+p_{15}\right)
$$

In Eqs. (8), (9), (9'), (9"), (9"') and Eq. (10) it is considered that $p_{j}=0$ if $j \leq 0$ or $j \geq 16$.

$a j$ ) If the unpaired user is from the area $a j, j=1,2, \ldots, 15$, pairing probability is

$$
P u(j)=p_{j} \cdot\left(p_{j-2}+p_{j-1}+p_{j}+p_{j+1}+p_{j+2}\right) .
$$

bj) The successful pairing probability after one rearrangement is

$$
\begin{aligned}
& \operatorname{Pr} 1(j)=p_{j} \cdot\left(p_{j-3}\right) \cdot\left\{1-\left[1-\left(p_{j-2}+p_{j-1}\right)\right]^{2 N-2}\right\}, \\
& \operatorname{Pr} 2(j)=p_{j} \cdot\left(p_{j-4}\right) \cdot\left\{1-\left[1-\left(p_{j-2}\right)\right]^{2 N-2}\right\}, \\
& \operatorname{Pr} 3(j)=p_{j} \cdot\left(p_{j+3}\right) \cdot\left\{1-\left[1-\left(p_{j+2}+p_{j+1}\right)\right]^{2 N-2}\right\}, \\
& \operatorname{Pr} 4(j)=p_{j} \cdot\left(p_{j+4}\right) \cdot\left\{1-\left[1-\left(p_{j+2}\right)\right]^{2 N-2}\right\}, \\
& \operatorname{Pr}(j)=\operatorname{Pr} 1(j)+\operatorname{Pr} 2(j)+\operatorname{Pr} 3(j)+\operatorname{Pr} 4(j) .
\end{aligned}
$$

As the number of connections, i.e. $N$, is greater in the state $\{2 N-1\}$, greater is probability that some user is situated in $4 \mathrm{~dB}$ neighbouring of the new call, i.e. pairing after rearrangement is more probable. This may be concluded from Eqs. (9), (9'), (9") and (9"') where greater values of $N$ cause greater values of $P r$.

cj) Probability that pairing may not be realized is

$P n(j)=p_{j} \cdot\left(p_{1}+\ldots+p_{j-5}+p_{j+5}+\ldots+p_{15}\right)$. 
If there is only one unpaired $\mathrm{OSC}$, total direct pairing probability $P u$ is

$$
P u=\sum_{j} P u(j)
$$

In the same state with one unpaired OSC, total pairing probability after one rearrangement $(\mathrm{Pr})$ is

$$
\operatorname{Pr}=\sum_{j} \operatorname{Pr}(j)
$$

and the total probability of impossible pairing in this case is

$$
P n=\sum_{j} P n(j)
$$

If pairing is realized without rearrangement, the probability of paired connections is $P u$. If pairing is realized using rearrangement, the probability of paired connections is $P u+P r$. The pairing gain due to rearrangement implementation is, therefore, $P r / P u$ and it is related to the state $\{2 N-1\}$. The total gain, i.e. loss decrease when pairing is impossible, is

$$
D=P\{2 N-1\} \cdot P r .
$$

\section{NUMERICAL EXAMPLES}

Example 1: The group of 8 channels is examined in the following analysis. In this cell there is the same number of users in each ring with the same attenuation. It means that call probabilities from each ring are equal,

$$
p_{1}=p_{2}=\ldots=p_{16}=\frac{1}{16} \text {. }
$$

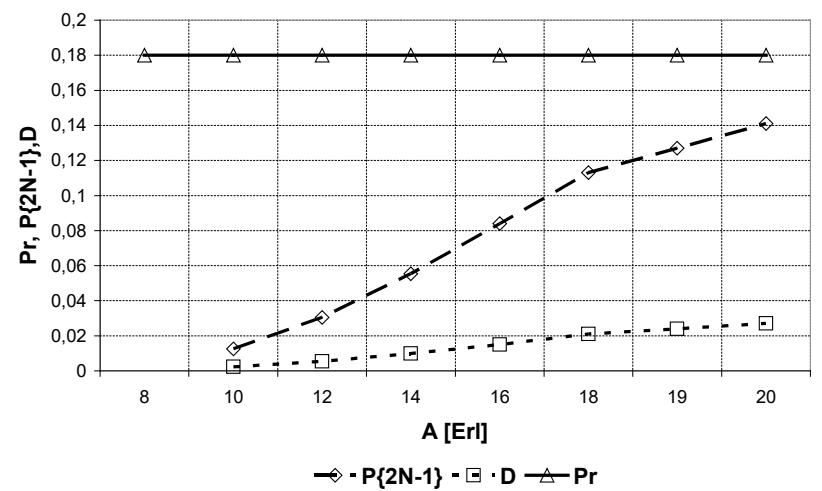

Figure 6 Pairing gain (loss decrease due to pairing inability) caused by rearrangement as a function of offered traffic for a system with 8 channels (16 OSCs)

Full line in Fig. 6 presents probability that rearrangement may be realized in the state $\{2 N-1\}$ to allow pairing in which signal power difference needed for paired users is not greater than $4 \mathrm{~dB}$. In this case, a pairing gain exists only in the state $\{2 N-1\}$. When considering the whole traffic process, total gain is expressed by the Eq. (14). The dependence of state probabilities on the offered traffic is presented by dashed line in Fig. 6. It is obvious that value of total gain, $D$, is several percents only for very great values of offered traffic, dotted line in Fig. 6.

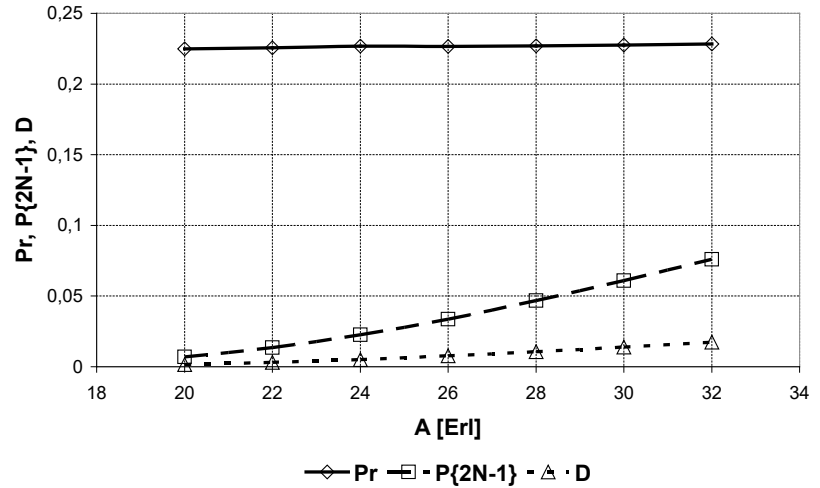

Figure 7 Pairing gain (loss decrease due to pairing inability) caused by rearrangement as a function of offered traffic for a system with 14 channels (28 OSCs)

Example 2: The results for the greater group of 14 channels are presented in Fig. 7. Comparing these results to the results from Fig. 6, it may be concluded that total pairing probability after one rearrangement $(P r)$ increases when the number of available traffic channels increases. In the same time the total gain $(D)$ when considering also the probability of the state $2 N-1(P\{2 N-1\})$ decreases when the number of channels increases. This last general conclusion for $D$ is based referencing the point where the offered traffic $A$ in Erlangs is equal to the number of available OSCs (16E in Fig. 6 and 28E in Fig. 7).

These two numerical examples present the trend, which is expected for system characteristics $\operatorname{Pr}$ and $D$ when the number of channels increases. But, in the same time $70 \%$ of total number of realized mobile systems, which are candidates for VAMOS implementation, have no more than 14 traffic channels in their practical realization, [24] and their characteristics correspond to the ones presented by Fig. 6 and Fig. 7.

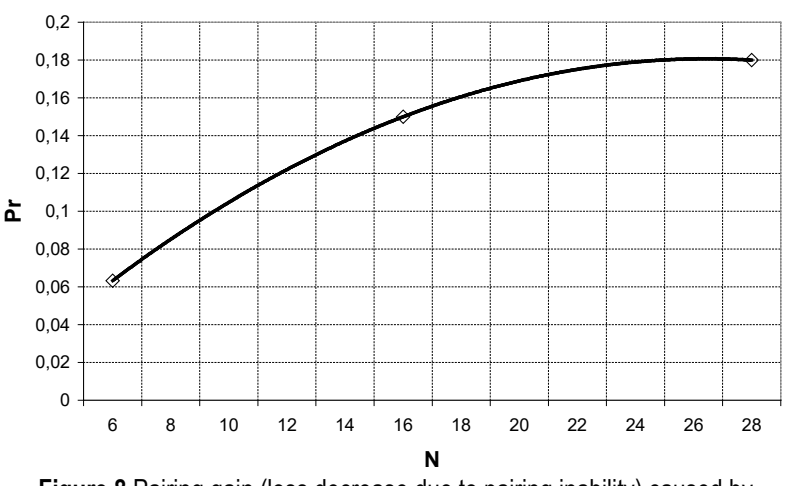

Figure 8 Pairing gain (loss decrease due to pairing inability) caused by rearrangement as a function of available number of channels

Fig. 8 presents the pairing probability change as the function of the number of channels when one rearrangement is realized in the state $\{2 N-1\}$. The conditions are the same as in Fig. 6 and Fig. 7, except for the changed number of channels. This graph verifies the conclusion that pairing probability after one connection rearrangement increases when the number of channels increases. 


\section{SIMULATION VERIFICATION}

Simulation program for the system with implemented VAMOS technique with rearrangement possibility is developed on the basis of already developed simulation programs for different mobile telephony systems, [25][27]. Simulation program is based on classic Monte Carlo method, [28], which we adjusted for solving our specific problem.

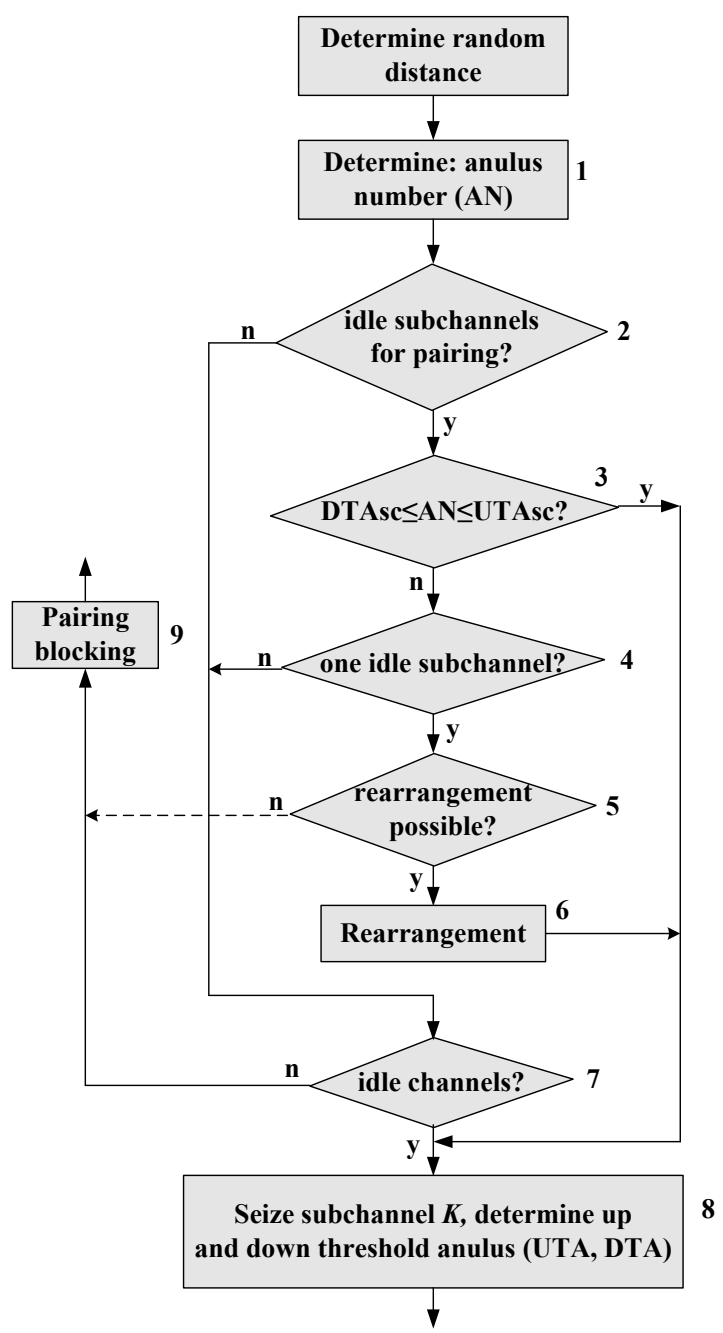

Figure 8 Supplement to the simulation program presented in [25], intended to the analysis of VAMOS system with rearrangement possibility

The starting point in our simulation has been simulation program from our previous research [25]. The part, which has now been added to the program from [19] to analyse VAMOS systems with implemented rearrangement, is presented in Fig. 8. In this simulation process we implemented algorithm with first pairing calls and then seizing one idle OSC in a completely idle timeslot (channel) (if there are no timeslots with only one idle OSC).

The first step in program additional part is to find the annulus where the user, who generates the request, is situated (block 1). Then it is tested whether there are timeslots with one idle OSC (subchannel). These timeslots are candidates for pairing (block 2). In the case that there are timeslots with one idle OSC, it is tested whether the newly generated request is located in an annulus, whose ordinal number (AN) is between the upper (UTAsc) and the lower threshold (DTAsc) of the annulus ordinal number, intended for pairing (block 3 ). If pairing may be performed in some timeslot, it is realized in the block 8 . The upper (UTA) and the lower annulus threshold number (DTA) are determined for an eventual later pairing with this newly generated request.

Rearrangement is performed when there is only one idle OSC in the whole simulated system and no completely idle channels. That is why it is checked in block 4 what the number of idle OSCs is. In the case that there is one idle OSC, it is investigated in block 5 whether annuli numbers of busy OSCs in the system allow rearrangement realization. In the case that rearrangement is possible, it is realized in block 6 (rearrangement is a complex process and therefore it is not presented in detail). After rearranging already realized requests, new request is paired in the block 8 with the unpaired request. In the case that rearranging is not possible (according to the conclusion in the block 5), the pairing loss counter is incremented in block 9 .

It is necessary to check in block 7 whether there are completely idle channels in the case that there are no idle OSCs ready for pairing (test in block 2) and if there is more than one idle OSC in the whole system (test in block 4). If such completely idle timeslot does not exist, call blocking due to pairing inability is recorded in block 9 .

\section{CONCLUSION}

VAMOS technique significantly increases traffic possibilities of the traffic channel group in a cell. Pairing inability of the last idle traffic subchannel (OSC) due to the too great power difference between two users - candidates for pairing prevents to achieve double traffic capacity increase comparing to classical model. Efficiency increase in the analyzed systems is the consequence of established connections rearrangement in the state when only one idle OSC exists. The greatest gain caused by rearrangement in relation to the system without rearrangement is achieved when allowed power difference between two paired users is small $(4 \mathrm{~dB})$. The important conclusion is that gain obtained by one rearrangement is the greatest in the periods of traffic overload, when this gain is the most important. The gain is greater in the greater channel groups. The connection seizing delay due to rearrangement is negligible and imperceptible to users. Finally, the results of computationally obtained loss values are verified in simulation.

The procedure for rearrangement realization is similar to the handover procedure in one BS cell when only the busy channel is changed (intra-cell handover), [29]. Although mutually similar for the realization, there are differences in reasons of rearrangement and intra-cell handover implementation in mobile systems. While intracell handover is applied in order to improve connection signal quality by changing the dedicated traffic channel to decrease the effect of interference and fading, [29], the rearrangement contributes to the better traffic characteristics (to the lower traffic loss). The connection signal quality in paired channels may be even slightly worse than if VAMOS is not applied. 


\section{Acknowledgements}

This paper is realized within the projects TR32051 and TR32007, which are cofinanced by Ministry of Education, Science and Technological Development of the Republic of Serbia.

\section{REFERENCES}

[1] Säily, M., Sébire, G., \& Riddington, E. (2011). GSM/EDGE Evolution and Performance. New York, John Wiley \& Sons. https://doi.org/10.1002/9780470669624.ch8

[2] Huang, W. (2010). Widely Linear MIMO MMSE Filter and Joint MLSE for VAMOS. Thesis for the degree of Master of Applied Sciences, University of Waterloo.

[3] Alcatel, L. (2012). GSM Voice Capacity Evaluation with VAMOS. Strategic White Paper.

[4] Perić, M., Jakšić, B., Aleksić, D., Randjelović, D., \& Stefanović, M. (2018). Outage Probability of Macrodiversity Reception in the Presence of Fading and Weibull CoChannel Interference. Technical Gazette, 25(2), 376-381. https://doi.org/10.17559/TV-20161227102847

[5] Xiaoqun, H. (2010). GBSS13.0 BSC6900 (V900R013C00) VAMOS Feature Description. Global Technical Support.

[6] (2013). Voice services over Adaptive Multi-user Orthogonal Sub channels, An Insight, Mobile Division TEC.

[7] Lebl, A., Mitić, D., Mileusnić, M., Matić, V., Markov, Ž., \& Marinković-Nedelicki, V. (2016). Analysis of Possibilities to Increase Traffic Capacity in GSM Systems. International Scientific Conference "Unitech 2016". November 18-19, Gabrovo, (Bulgaria), II-57-II-59.

[8] Enache, B. (2015, September). Transmission Techniques for VAMOS GSM in Downlink. Acta Univerversitatis Technical Series, vol. LXVI. https://doi.org/10.1515/aucts-2015-0025

[9] Budura, G. \& Balint, C. (2014). Pairing Strategy for VAMOS Downlink Transmission. $11^{\text {th }}$ International Symposium on Electronics and Telecommunications (ISETC). https://doi.org/10.1109/ISETC.2014.7010768

[10] Balint, C. \& Budura, G. (2014). System Model for Performance Evolution VAMOS Downlink Transmission. $14^{\text {th }}$ International Conference on Optimization of Electrical and Electronic Equipment OPTIM 2014. https://doi.org/10.1109/OPTIM.2014.6850933

[11] Ruder, M., Meyer, R., Kalveram, H., \& Gerstacker, W. (2012). Radio resource allocation for OSC downlink channels. $1^{\text {st }}$ IEEE International Conference on Communications in China Workshops (ICCC). https://doi.org/10.1109/ICCCW.2012.6316464

[12] Ruder, M. A. (2014). User Pairing for Mobile Communication Systems with OSC and SC-FDMA Transmission. Dissertation. Friedrich-AlexanderUniversität.

[13] Mitić, D., Lebl, A., \& Markov, Ž. (2018). Calculation of Call Unpairing Probability in GSM Cell Using VAMOS Technics. Ingeniería e investigación, 38(3), 50-58. https://doi.org/10.15446/ing.investig.v38n3.67523

[14] Mitić, D., Lebl, A., Markov, Ž., \&Stanić, M. (2018). Comparison of two methods for subchannel seizure in GSM cell using VAMOS technics, Journal of Electrical Engineering, 69(3), 205-211. https://doi.org/10.2478/jee-2018-0027

[15] Jovanović, P., Mileusnić, M., Lebl, A., Mitić, D., \& Markov, Ž. (2014). Calculation of the Mean Output Power of Base Transceiver Station in GSM. Automatika, 55(2), 182-187. https://doi.org/10.7305/automatika.2014.06.373

[16] Su, X., Wang, Y., Choi, D., Kim, P., \& Choi, C. (2017). Channel allocation and power control schemes for cross-tier
3GPP LTE networks to support multimedia applications. Multimedia Tools and Applications, 76(24), 25875-25891. https://doi.org/10.1007/s11042-016-4320-3

[17] Ebersprächer, J., Vögel, H.-J., \& Bettstetter, Ch. (1999). GSM, Switching, Services and Protocols. New York, John Wiley \& Sons. https://doi.org/10.1002/0470841745

[18] ITU-T: Recommendation E.721 - Network grade of service parameters and target values for circuit-switched services in the evolving ISDN, (1999, May).

[19] ITU-T: Recommendation E.671 - Post-selection delay in PSTN/ISDN networks using Internet telephony for a portion of the connection, (2000, March).

[20] Heine, G. (1999). GSM Networks: Protocols, Terminology and Implementation. London, Artech House. http://ik4hdq.net/doc/testi/gsm networks.pdf

[21] $3^{\text {rd }}$ Generation Partnership Project. (2011). Technical Specification Group GSM/EDGE Radio Access Network; Radio subsystem link control (Release 10). 3GPP TS45.008 $\mathrm{v} 10.0$

[22] Das, S. K., Krishnamoorthz, A., \& Muppirisetty, L. S. (2014). EP 2689551 B1, Proprietor Telefonaktiebolaget L. M. Ericsson, Stockholm.

[23] Beneš, V. E. (1965). Mathematical Theory of Connecting Networks and Telephone Traffic, New York, Academic Press. eBook ISBN: 9780080955230.

[24] Colombi, D., Thors, B., Persson, T., Wirén, N., Larsson, L.E., Jonsson, M., \& Törnevik, C. (2013). Downlink power distribution for $2 \mathrm{G}$ and $3 \mathrm{G}$ mobile communication networks, Radiation Protection Dosimetry, 157(4), 477-487. https://doi.org/10.1093/rpd/nct169

[25] Mileusnić, M., Popović, M., Lebl, A., Mitić, D., \& Markov, Ž. (2014). Influence of Users' Density on the Mean Base Station Output Power. Elektronika i Elektrotechnika, 20(9), 74-79. https://doi.org/10.5755/j01.eee.20.9.5418

[26] Šuh, T., Lebl, A., Mitić, D., \& Markov, Ž. (2014). Use of Computer Simulation in Estimation of GSM Base Station Output Power. Acta Polytechnica Hungarica, 11(6), 129142. https://doi.org/10.12700/APH.11.06.2014.06.8

[27] Mileusnić, M., Jovanović, P., Popović, M., Lebl, A., Mitić, D., \& Markov, Ž. (2014). Influence of Intra-cell Traffic on the Output Power of Base Station in GSM, Radioengineering, 23(2), 601-608. https://www.radioeng.cz/ fulltexts/2014/14_02_0601_0608.pdf

[28] Rubinstein, R.Y., Kroese, D.P. (2017). Simulation and the Monte Carlo Method. New York, $3^{\text {th }}$ Edition, John Wiley \& Sons. https://doi.org/10.1002/9781118631980

[29] López-Pérez, D., Valcarce, A., Ladányi, Á., de la Roche, G., \& Zhang, J. (2010). Intracell Handover for Interference and Handover Mitigation in OFDMA Two-Tier MacrocellFemtocell Networks. EURASIP Journal on Wireless Communications and Networking, 1-15, https://doi.org/10.1155/2010/142629 


\section{Contact information:}

Aleksandar LEBL, PhD

(Corresponding author)

IRITEL a.d.

Batajnički put 23, 11080 Belgrade, Serbia

lebl@iritel.com

Dragan MITIĆ, PhD

IRITEL a.d.

Batajnički put 23, 11080 Belgrade, Serbia

mita@iritel.com

Žarko MARKOV, PhD, Prof.

IRITEL a.d.

Batajnički put 23, 11080 Belgrade, Serbia

zmarkov@iritel.com

Mihailo STANIĆ, PhD

IRITEL a.d.

Batajnički put 23, 11080 Belgrade, Serbia

mihailo@iritel.com

Mladen MILEUSNIĆ, PhD

IRITEL a.d.

Batajnički put 23, 11080 Belgrade, Serbia

mladenmi@iritel.com 